\title{
Levantamento de insetos aquáticos: auxílio para adoção de práticas sustentáveis em propriedades leiteiras
}

A alta produtividade da pecuária leiteira pode afetar a qualidade da água dos recursos hídricos, por meio da contaminação de nutrientes e de substâncias químicas despejadas diretamente nos seus cursos d'água. A vegetação ciliar que compõe as Áreas de Preservação Permanente (APP) destes ecossistemas aquáticos também é prejudicada devido a ocupação do gado leiteiro em suas margens. Este artigo se propõe a destacar algumas reflexões teóricas em torno de como os resultados obtidos por meio do levantamento dos insetos aquáticos nos ambientes lóticos inseridos dentro das propriedades leiteiras podem contribuir para os desafios da busca pela sustentabilidade. Para tanto foi realizada uma revisão da literatura do tipo narrativa, para a qual foram selecionadas 21 publicações nas bases Periódicos CAPES, Scielo, Directory of Open Access Journals (DOAJ), Science.com e da Embrapa - Pesquisa Agropecuária, através dos quais emergiram três categorias de análise: a) Mercado leiteiro e a globalização; b) processo de Governança na pecuária leiteira; c) insetos aquáticos como bioindicadores para atingir a sustentabilidade ambiental. As categorias analisadas neste trabalho ressaltam a importância da produção leiteira para a economia local e global, além da sua relevância social que deve ser fortalecida através do processo de governança. As análises também evidenciaram a necessidade da implantação da sustentabilidade dentro das propriedades leiteiras, a fim de proporcionar uma melhora da qualidade ambiental dos recursos naturais, gerando assim um aperfeiçoamento da qualidade do leite ofertada pelo produtor rural.

Palavras-chave: Sustentabilidade; Governança; Globalização; Bioindicadores.

\section{Survey of aquatic insects: aid for the adoption of sustainable practices in dairy farms}

\begin{abstract}
High productivity of dairy farming may affect water quality of resources through contamination of nutrients and chemicals dumped directly on their watercourses. The riparian vegetation that makes up the Permanent Preservation Areas (APP) of these aquatic ecosystems is also impaired due to the occupation of the dairy cattle on its shores. This article aims to highlight some theoretical reflections on how the results obtained through the survey of the aquatic insects in the lotic environments within dairy farms can contribute to the challenges faced by the pursuit of sustainability. To this end, a literature review of the narrative type was carried out, for which 21 publications were selected in the Periodical databases CAPES, Scielo, Directory of Open Access Journals (DOAJ), Science.com and Embrapa - Agricultural Research, and three categories of analysis emerged: a) Dairy market and globalization; B) Governance process in dairy farming; c) Aquatic insects as bioindicators for achieving environmental sustainability. The categories analyzed in this paper highlight the importance of dairy production for the local and global economy, as well as its social relevance that must be strengthened through the governance process. The analysis also showed the need to implement sustainability in dairy farms in order to provide an improvement in the environmental quality of natural resources, thus generating an improvement of milk quality offered by the rural producer.
\end{abstract}

Keywords: Sustainability; Governance; Globalization; Bioindicators.

Topic: Desenvolvimento, Sustentabilidade e Meio Ambiente

Reviewed anonymously in the process of blind peer.

Bruna Ehlert (iD)

Universidade do Vale do Taquari, Brasil http://lattes.cnpq.br/6461716175156655

http://orcid.org/0000-0003-3308-2914

bruna.ehlert@hotmail.com

\section{Claudete Rempel (iD)}

Universidade do Vale do Taquari, Brasil

http://lattes.cnpq.br/8340497822227462

http://orcid.org/0000-0001-8573-0237

crempel@univates.br

\section{Marina Schmidt Dalzochio (iD)}

Universidade do Vale do Taquari, Brasil

http://lattes.cnpq.br/8400882066099257

http://orcid.org/0000-0001-9241-921X

mahsdalzochio@gmail.com
Received: 06/12/2019

Approved: 20/01/2020

\section{Referencing this:}

EHLERT, B.; REMPEL, C.; DALZOCHIO, M. S.. Levantamento de insetos aquáticos: auxílio para adoção de práticas sustentáveis em propriedades leiteiras. Revista Ibero Americana de Ciências Ambientais, v.11, n.1, p.341-350, 2020. DOI: http://doi.org/10.6008/CBPC2179-6858.2020.001.0031 


\section{INTRODUÇÃO}

Este artigo faz parte do projeto de doutoramento do Programa de Pós-Graduação em Ambiente e Desenvolvimento, da Universidade do Vale do Taquari - UNIVATES. Tem como proposta avaliar a relação da riqueza, abundância e a composição de insetos aquáticos com os estados de conservação da cobertura vegetal das Áreas de Preservação Permanente (APP), com a finalidade de, por meio dos dados obtidos, aperfeiçoar um indicador de sustentabilidade ambiental ao longo da microbacia do Arroio da Seca, nos municípios de Colinas e Imigrante, bacia hidrográfica do Taquari-Antas, porção Taquari.

No contexto da atual fase de investigação, este artigo pretende destacar algumas reflexões teóricas em torno de como os resultados obtidos por meio do levantamento dos insetos aquáticos nos ambientes lóticos inseridos dentro das propriedades leiteiras podem contribuir para a implantação da sustentabilidade, dos desafios impostos pela globalização, para a governança local e a participação pública dos produtores rurais locais.

As atividades antrópicas, voltadas à agricultura, ameaçam a qualidade dos recursos hídricos, dentre estas atividades a produção leiteira destaca-se devido a poluição pelo uso de produtos químicos, pelo aumento das taxas de lotação e pela contaminação fecal (WILLIS, 2001). Sendo que a ocupação do gado leiteiro na APP provoca o desmatamento da vegetação ciliar nas bacias hidrográficas devido ao pisoteio e a pastagem (BUENDIA et al., 2016). Neste aspecto é aconselhável que os produtores rurais adotem práticas visando também a utilização planejada dos recursos naturais, buscando o equilíbrio entre a atividade exercida e a conservação do ambiente.

Entre os organismos encontrados nos ambientes lóticos associados às APP, estão os insetos aquáticos, sendo estes utilizados como organismos bioindicadores devido ao alto grau de sensibilidade aos impactos ambientais (MORENO et al., 2009). Considerando o alto potencial dos insetos aquáticos em resposta aos diversos impactos ambientais e a importância da preservação da vegetação ciliar, identificar organismos, capazes de indicar o nível de qualidade destes ambientes aquáticos se mostra uma ferramenta importante no processo de conservação ambiental.

Diante do grande potencial dos insetos aquáticos como bioindicadores da qualidade dos ecossistemas lóticos das propriedades rurais leiteiras (BRACCIA et al., 2006), emerge a sustentabilidade ambiental, sendo esta fundamental para a redução das problemáticas tanto ambientais, como dos impactos socioeconômicos nestes ambientes. Devido aos inúmeros impactos ambientais causados pelas atividades agrícolas, a atenção para sustentabilidade nestes ambientes torna-se essencial, onde o espaço rural passa a ser o principal agente ativo frente a conservação e proteção dos recursos naturais, sendo que o conhecimento da exploração dos limites possíveis destes ecossistemas é um fator primordial para o desenvolvimento de ambientes sustentáveis (ALVES et al., 2015).

Além de traçar novos caminhos para a sustentabilidade dentro das propriedades produtoras de leite, as respostas adquiridas por meio de levantamentos de insetos aquáticos bioindicadores do estado de conservação destes recursos hídricos e de suas APP, também permitirão que ocorra o processo de 
governança e participação social dentro da pecuária leiteira. Assim, este artigo objetiva refletir, por meio de análise de uma revisão narrativa, acerca de como os resultados obtidos por meio do levantamento dos insetos aquáticos nos ambientes lóticos inseridos dentro das propriedades leiteiras podem contribuir para os desafios da busca da sustentabilidade.

\section{METODOLOGIA}

No presente estudo foi realizada uma revisão da literatura do tipo narrativa, sobre os aspectos da produção leiteira, buscando maiores informações sobre esta atividade produtiva. Segundo Knechtel (2014) a pesquisa bibliográfica possibilita a elaboração do conhecimento através da leitura. Para Creswel (2010) o aparato bibliográfico proporciona a informação de estudos já realizados, possibilitando a informação de variadas referências.

Foram pesquisados artigos científicos em diferentes bases de dados: Scielo, Periódicos CAPES, Directory of Open Access Journals (DOAJ), Science.gov e Pesquisa Agropecuária Embrapa. Sendo utilizados como critérios para a coleta artigos que continham a expressão 'leiteira', 'leite' e 'lácteos' no título, ou 'gado' e 'pastagem' em qualquer parte do artigo. Foram considerados os trabalhos publicados em português, espanhol ou inglês. Após foi realizada uma triagem dos trabalhos mais relevantes, onde foram descartados os estudos que não apresentavam conteúdos pertinentes ao objetivo da pesquisa.

\section{DISCUSSÃO TEÓRICA}

No total foram avaliados 21 artigos científicos, a partir da leitura deles, emergiram três categorias de análise: a) Mercado leiteiro e a globalização; b) O processo de Governança na pecuária leiteira; c) Insetos aquáticos como bioindicadores para atingir a sustentabilidade ambiental.

\section{Mercado leiteiro e a globalização}

A produção de leite no país passou por inúmeras transformações na década de 1990, devido às mudanças geradas na economia mundial impostas pela globalização, como o fim da atuação do Estado na construção do preço, o começo do mercado doméstico, o êxito do Plano Real, o fim do Programa de Tíquete do Leite, onde este tinha o Estado como o grande comprador do produto final e a implantação do Plano Nacional de Qualidade do Leite (BREITENBACH et al., 2011). A cadeia produtiva de leite tem sofrido várias modificações para atender as exigências do mercado consumidor, onde a globalização influencia diretamente o mercado leiteiro que necessita cada vez mais de melhores planejamentos para incentivar o seu desenvolvimento competitivo (ISERMEYER et al., 2003). Neste sentido se intensificam as exigências aos produtores rurais na qualidade da sua matéria prima, havendo cada vez uma maior necessidade destes trabalhadores rurais adotarem novas tecnologias que priorizem a qualidade ambiental e consequentemente a melhora na qualidade de seus produtos, o estudo publicado por Rodrigues et al. (2006) revela que a inclusão tecnológica dentro da pecuária leiteira resulta em sinais positivos referentes aos impactos sócios-ambientais dentro das propriedades. 
Diante deste contexto cabe ressaltar que o Brasil é um dos maiores produtores de leite do mundo, sendo que a maioria da produção agropecuária nacional é representada pela agricultura familiar, onde a produção brasileira de leite cresceu $55 \%$ na última década e, atualmente, o país é o quinto maior produtor mundial, atrás dos países que formam a União Europeia, a Índia, os Estados Unidos e a China (NERES et al., 2018). Portanto no cenário econômico nacional o Brasil baseou-se no seguimento apenas voltado para a exploração individual e na concentração dos meios de produção da agricultura, não valorizando a importância que a agricultura familiar possui na agropecuária. Sendo que está se destaca na produção de alimentos, na economia através da distribuição de renda e geração e oferta de empregos (OLIVEIRA et al., 2012).

O Vale do Taquari destaca-se no mercado leiteiro, sendo a produção de leite como a principal fonte de renda de muitas famílias. Porém a agricultura familiar encontra inúmeros obstáculos como a carência de infraestrutura e de políticas públicas (MENEGHATTI et al., 2017), causando muitas vezes o empobrecimento e a expulsão dos agricultores familiares do campo.

O processo de globalização vem acompanhado da modernização e do desenvolvimento das forças produtivas, onde o capitalismo mundial provoca uma fragmentação territorial devido a globalização, nos anos 2001/2002 o mercado leiteiro enfrentou uma crise gerada pela diminuição nos preços recebidos pelos produtores, onde vários deixaram a atividade e como consequência a produção láctea diminuiu (MORAES et al., 2003). Apesar das dificuldades impostas pela globalização aos produtores rurais, a produção leiteira vem crescendo, onde o mercado está cada vez mais exigente em relação à qualidade do leite, devido à percepção atual da sociedade sobre o papel exercido pelos alimentos em relação a saúde dos consumidores (PAIXÃO et al., 2014). Sendo a baixa qualidade do leite atribuída a diversos fatores como falta de higiene na ordenha, dificuldades na manutenção e limpeza de equipamentos e refrigeração ineficaz (NERO et al., 2005).

Embora a qualidade do leite destaca-se como o fator de essencial interesse para o mercado consumidor, as preocupações com a sustentabilidade ambiental dentro da pecuária leiteira também têm recebido uma importante relevância, o estudo feito por Rempel et al. (2012) salientam a importância da sustentabilidade dentro da cadeia produtiva da leiteira. Dentro deste contexto a execução de práticas sustentáveis para a melhoria da qualidade ambiental torna-se primordial para a sustentabilidade ambiental e a minimização de impactos ambientais. Nesse sentido, a adoção de práticas sustentáveis favorece os parâmetros produtivos das propriedades, atingido assim uma melhora na qualidade do leite, favorecendo o agronegócio e a economia familiar (DOMENICO et al., 2017).

\section{O processo de Governança na pecuária leiteira}

Diante da importância econômica da pecuária leiteira para o país e mais especificamente, neste estudo, para o Vale do Taquari, a melhoria nos níveis de bem-estar social e de qualidade de vida da agricultura familiar deve ser valorizada através da intervenção da mobilização e valorização dos recursos humanos, institucionais, econômicos, culturais e ambientais. Porém para que os aspectos citados anteriormente sejam alcançados dentro da atividade leiteira, deve haver uma maior articulação dos produtores rurais ao sistema 
estatal no processo político e formação de políticas públicas (YAMAGUCHI et al., 2001).

Tendo em vista que na sociedade há diferentes padrões de adesão a determinados padrões tecnológicos, muitas vezes ocorre a exclusão de alguns agentes, onde torna-se imprescindível a participação dos sujeitos locais para o conhecimento a respeito de tais processos e os impactos nas avaliações de políticas públicas vigentes ou em sua formulação (SOUZA et al., 2013). Segundo Domenico et al. (2017) o ambiente competitivo do mercado leiteiro gerou transformações na postura dos agentes locais e na modificação da estrutura de governança, sendo coordenada pelo mercado. O estudo realizado por Acosta et al. (2017) destaca que os critérios de relação entre os produtores e o mercado envolvem a quantidade de leite produzida e a qualidade deste, além do preço, sendo constatado que o seguimento da relação envolve a especificidade locacional e temporal do ativo, na frequência, na ausência de oportunismo, na reputação e confiança (adimplência do comprador) e também da lealdade estabelecida entre as partes. Diante deste contexto, para os produtores, quanto maior a qualidade do leite, melhor será o preço de venda e o poder de renegociação (ACOSTA et al., 2017).

No entanto, ao se tratar da governança dentro da pecuária leiteira, esta quando executada, poderá proporcionar resultados positivos como a melhora da qualidade ambiental das propriedades rurais e consequentemente uma melhora na qualidade do leite, valorizando assim mais o produto frente ao mercado. No processo de governança o ambiente organizacional formado pelo conjunto de organizações criadas para dar suporte a um determinado Sistema Agroindustrial, são de fundamental importância dentro da cadeia produtiva. Estas organizações possuem o objetivo de defender interesses dos produtores, provocar mudanças institucionais, reclamar o surgimento de novas políticas públicas (OLIVEIRA et al., 2012). Neste sentido a prática da governança possibilita que os produtores tenham acesso às informações, onde muitos sujeitos locais sofrem uma grande carência ao acesso do conhecimento de suas atividades produtivas. Segundo Casali et al. (2012), os produtores leiteiros apresentaram um grau de incerteza em relação ao acesso à informação, sendo que os sujeitos que possuem um maior conhecimento sobre o mercado e suas operações, possuem vantagens em relação a outra parte que não possui acesso à informação, propiciando assim muitas vezes atitudes oportunistas do mercado sobre os produtores.

Neste contexto os novos caminhos para a sustentabilidade, dentro da pecuária leiteira, por meio de práticas ambientais que visam à conservação dos recursos hídricos, como de suas APP e do ecossistema, podem ser traçados através do processo de governança. Segundo Campos et al. (2010) a governança possibilita soluções teóricas e práticas estabelecendo uma relação entre o nível governamental e as demandas sociais, gerindo os diferentes interesses existentes.

Diante do cenário global da pecuária leiteira, tanto na economia do mercado, como de suas práticas produtivas prejudiciais ao meio ambiente o conceito de governança torna-se fundamental de ser adotado, pois a noção do poder social dos atores, neste caso os produtores rurais devem ser valorizados nas relações entre o Estado, a sociedade civil e o mercado. Neste sentido, a participação ativa dos produtores rurais na busca de medidas mitigadoras para a melhora da qualidade ambiental onde dá-se a produção leiteira o processo de governança possibilitará a informação e o conhecimento necessário para a realização de um 
bom gerenciamento sustentável da atividade leiteira. Como afirmam Lopes et al. (2016), um aspecto negativo que limita o desenvolvimento da gestão dentro da pecuária leiteira é o despreparo dos produtores de leite, que muitas vezes não possuem conhecimento e disposição suficientes para realizar uma boa atividade gerencial dentro de suas propriedades. Sendo que a limitação de fundos financeiros e a baixa escolaridade dos profissionais que trabalham no campo são condições fortemente influenciáveis (ABRAHÃO et al., 2015). Esses comportamentos comprometem o desenvolvimento da atividade leiteira e interferem negativamente na gestão ambiental e econômica (LOPES et al., 2016).

Compreender o impacto ambiental causado pela falta de planejamento ambiental dentro da pecuária leiteira é fundamental para assegurar que os resultados mais satisfatórios sejam atingidos de modo que, segundo Silva et al. (2015), possam contribuir para que a pecuária leiteira se torne economicamente mais atrativa. Devido à crise econômica dos últimos anos que atinge o agronegócio de leite, a perda de atratividade do setor tem sido uma realidade em propriedades tecnologicamente e ambientalmente menos evoluídas (LIMA FILHO et al., 2016).

Diante do exposto a crise política e econômica a qual o país está inserido é um elemento de forte impacto na atividade leiteira, onde o processo de governança torna-se uma ferramenta fundamental, segundo Lemos et al. (2003) a heterogeneidade da pecuária leiteira impõe grandes desafios na criação e execução de políticas na esfera regional voltadas para a modernização e diminuição das desigualdades frente aos produtores. Assim sendo, para que o desenvolvimento das atividades na pecuária leiteira seja positivo, estas necessitam de práticas planejadas para enfrentarem o mercado e a globalização para se manterem competitivos.

\section{Insetos aquáticos como bioindicadores para atingir a sustentabilidade ambiental}

Os recursos hídricos estão entre os ecossistemas mais ameaçados no mundo, sendo que a intensa urbanização provoca o aumento demasiado da demanda pela água e provoca alterações de ordem física, química e biológica nestes ambientes (TUNDISI, 2008). Devido este fato estes ambientes têm sido avaliados por meio do biomonitoramento, que possui como objetivo monitorar os impactos em ambientes de água doce através de macroinvertebrados aquáticos (BUSS et al., 2003). Os organismos supracitados são sensíveis e vulneráveis a mudanças antrópicas do seu habitat, apresentando, assim, respostas devido aos impactos físicos e químicos (MANGADZE et al., 2016).

Entre os macroinvertebrados aquáticos, os insetos aquáticos destacam-se em comparação com outras comunidades biológicas para o biomonitoramento por possuírem inúmeras vantagens. Dentre as quais, inclui-se sensibilidade referente à poluição, ciclo de vida relativamente longo, mobilidade reduzida, bem como, facilidade de coleta e identificação (BALDERAS et al., 2016). Deste modo, estes organismos aquáticos são considerados como um complemento essencial nas análises de água, contribuindo para a avaliação ecológica desses ecossistemas (SANTOS et al., 2015).

Nos ecossistemas aquáticos, os grupos de invertebrados mais sensíveis e intolerantes à poluição aquáticos estão os insetos aquáticos, pois estes necessitam de elevadas concentrações de oxigênio dissolvido 
na água (GOULART et al., 2003). O uso destes organismos é essencial para a avaliação da qualidade de recursos hídricos, não somente para mensurar a poluição das águas, mas também pelo fato da alta sensibilidade que possuem frente às mudanças de habitats, constituindo um grupo de importantes bioindicadores da qualidade da água (RESH et al., 1993).

Inúmeros estudos utilizaram comunidades de insetos de água doce para o biomonitoramento da qualidade da água e da degradação ambiental (RIOS et al., 2006; DAVIES et al., 2010; GONZALO et al., 2013; LI et al., 2019). Variadas pesquisas ressaltam a importância da presença dos insetos aquáticos das ordens Ephemereoptera, Plecoptera e Trichoptera, sendo estes considerados ótimos indicadores biológicos devido à sua alta sensibilidade a mudanças antrópicas (HAUER et al., 2007). Onde ambientes urbanizados apresentam uma menor riqueza e abundância destes organismos intolerantes (CUFFNEY et al., 2010).

Contudo, estudos sobre o biomonitoramento de insetos aquáticos relacionados com nas APP em locais com a presença do gado leiteiro têm sido investigados (BRACCIA et al., 2006; RANGANATH et al., 2009). Conforme Braccia et al. (2007) o acesso do gado nas APP pode resultar em uma série de impactos na ecologia aquática dos macroinvertebrados, devido a entrada de sedimentos, além de inúmeros fatores relacionados à qualidade da água.

Diante do exposto, ao refletirem as condições ambientais adversas, estes organismos auxiliam na deteç̧ão dos impactos negativos, contribuindo para um diagnóstico ambiental da qualidade ecológica da água (MOURA-SILVA et al., 2016). O conhecimento das comunidades de invertebrados nos ecossistemas aquáticos pode contribuir para o planejamento de ações voltados à conservação da biodiversidade e para a sustentabilidade ambiental (BARBOLA et al., 2011). Neste sentido em decorrência das consequências da deterioração ambiental causada pela pecuária leiteira nos ambientes lóticos é de grande relevância a atenção para análise de meios que venham subsidiar a preservação e a sustentabilidade destes ambientes naturais.

Devido a atual economia global e os diversos usos da água é um desafio crucial equilibrar as necessidades sociais e ecológicas para a sustentabilidade ambiental (GEIST et al., 2016). As respostas fornecidas através do levantamento dos insetos presentes nos ambientes lóticos impactados devido as alterações antrópicas servem para guiar a gestão local e para a criação de estratégias para a conservação da bacia hidrográfica (BRIED et al., 2016; MEYER et al., 2015).

\section{CONCLUSÕES}

Esta pesquisa, e consequentemente a aplicação dos resultados da mesma, contribuirá com a melhoria da qualidade ambiental, gerando condutas adequadas do produtor rural frente a sua produção, pois melhorando a qualidade ambiental, ocorre a melhora das APP, das nascentes e dos cursos d'água, muitas vezes utilizados para dessedentação animal, o que também contribuirá para a qualidade do leite. Sendo a qualidade do leite essencial para a economia do Vale do Taquari- RS, que por meio dessas ações, pode vir a se tornar um polo de referência na qualidade ambiental de propriedades produtoras do leite.

Portanto o levantamento da vegetação ciliar das APP relacionada aos insetos aquáticos neste projeto é de grande valia para diagnosticar e melhorar a situação ambiental das propriedades leiteiras, auxiliando 
assim através da informação os produtores rurais sobre a situação em que se encontram as suas terras, contribuindo de forma positiva para a criação de objetivos voltados à governança. Colaborando também para o processo de desenvolvimento local, através da democracia participativa entre o setor público, o setor privado e a comunidade para a identificação dos problemas e das competências locais, bem como, na elaboração e aplicação das atividades. Sendo que o desenvolvimento local é de fundamental relevância para a governança, sendo que este formado pela combinação de esforços tanto internos como externos, governamentais e não governamentais, públicos e privados. Dessa forma, o desenvolvimento local sustentável não ocorre sem a participação ativa da população local.

Além disso, a diversidade e riqueza encontrada serão correlacionadas com a diversidade de insetos aquáticos e da qualidade da água, pois se sabe que o principal motivo de preservação das APP é a manutenção da qualidade dos mananciais hídricos bem como para evitar erosão e assoreamentos, no entanto, nas propriedades produtoras de leite da região em estudo, estas áreas são muitas vezes utilizadas pelo gado para acesso aos recursos hídricos, contaminando a água com dejetos e também, devido à pressão de pastejo e pisoteio do local, diminuindo a diversidade e dificultando a regeneração natural dessas áreas. 0 acesso a este conhecimento por intermédio desta pesquisa, constitui-se em um fator estratégico para o alcance do desenvolvimento local sustentável dentro da pecuária leiteira, contribuindo assim para o desenvolvimento do capital humano.

A compreensão dos efeitos causados pela pecuária leiteira na diversidade de insetos aquáticos presentes nos ambientes lóticos dessas propriedades é de fundamental importância para a conservação da biodiversidade, assim como para o desenvolvimento de insetos aquáticos como indicadores de sustentabilidade ambiental. O levantamento da biodiversidade destes organismos associados aos impactos da atividade leiteira é determinante para o sucesso futuro das decisões destinadas para a sustentabilidade.

A relação do produtor rural com o meio acadêmico possibilitará troca de saberes e experiências, ou seja, a aquisição de conhecimento para ambos. Sendo que muitas vezes há uma grande carência de informações dos atores locais em relação ao planejamento, execução e acompanhamento das iniciativas para a criação de atividades produtivas inovadoras, onde há uma necessidade da atuação de um agente externo intermediador da formação e educação da comunidade com vistas ao desenvolvimento sustentável.

Através deste projeto de tese que está em andamento se espera que sejam detectados os pontos positivos e negativos das propriedades estudadas, e que estes resultados permitam aos produtores agirem sobre as questões possíveis de mudanças para melhoria da qualidade ambiental de suas terras, para que a sustentabilidade social, ambiental, econômica seja criada e desenvolvida diante das suas atividades.

\section{REFERÊNCIAS}

ABRAHÃO, R. F.; TERESO, M. J. A.; GEMMA, S. F. B.. A Análise Ergonômica do Trabalho (AET) aplicada ao trabalho na agricultura: experiências e reflexões. Revista Brasileira de Saúde Ocupacional, v.40, n.131, p.88-97, 2015.

ACOSTA, D. C.; SOUZA, J. P.. Estratégias de Organização da Cadeia do Leite no Paraná. Revista Ibero-Americana de
Estratégia, v.16, n.2, 2017.

ALVES, M. F. A.; RIBEIRO, S. N.; MELO, F. J. S.; ALVES, L. R. A.; VIEIRA, Z. C.. Avaliação da qualidade do solo em agroecossistemas na zona rural de São Bentinho-PB. Revista Verde de Agroecologia e Desenvolvimento Sustentável, v.10, n.5, 2015 
BALDERAS, E. C. S.; GRAC, C.; EQUILLE, L. B.; HERNANDEZ, M. A. A.. Potential application of macroinvertebrates indices in bioassessment of Mexican streams. Ecol. Indic., v.61, v.61, n.2, p.558-567, 2016.

BARBOLA, I. F. et. al. Avaliação da comunidade de macroinvertebrados aquáticos como ferramenta para o monitoramento de um reservatório na bacia do rio Pitangui, Paraná, Brasil. Série Zoologia, v.101, n.1-2, p.15-23, 2011.

BRACCIA, A.; VOSHELL, J.. Benthic macroinvertebrate responses to increasing levels of cattle grazing in Blue Ridge Mountain streams, Virginia, USA. Environ. Monit. Assess., v.131, n.1-3, p.185-200, 2007.

BRACCIA, A.; VOSHELL, J. R.. Benthic macroinvertebrate fauna in small streams used by cattle in the Blue Ridge mountains, Virginia. Northeast. Nat., v.13, n.2, p.269-286, 2006.

BREITENBACH, R.; SOUZA, R. S.. Caracterização de mercado e estrutura de governança na cadeia produtiva do leite na região noroeste do Rio Grande do Sul. Organizações Rurais \& Agroindustriais, Lavras, v.13, n.1, 2011.

BRIED, J. T.; SIEPIELSKI, A. M.; DVORETT, D.; JOG, S.. Species residency status affects model selection and hypothesis testing in freshwater community ecology. Freshwater Biology, v.61, n.9, 2016.

BUENDIA, C.; BUSSI, G.; TUSET, J.; VERICAT, D.. Effects of afforestation on runoff and sediment load in an upland Mediterranean catchment. Science Of The Total Environment, v.540, p.144-157, 2016.

BUSS, D. F.; BAPTISTA, D. F.; NESSIMIAN, J. L.. Bases conceituais para a aplicação de biomonitoramento em programas de avaliação da qualidade da água de rios. Caderno Saúde Pública, Rio de Janeiro, v.19, n.2, 2003.

CAMPOS, V. N. O.; FRACALANZA, A. P.. Governança das águas no Brasil: conflitos pela apropriação da água e a busca da integração como consenso. Rev. Ambiente \& Sociedade, Campinas, v.13, n.2, 2010.

CASALI, M. S.; MARION FILHO, P. J.. Custos de transação e governança na produção de leite em Cruz Alta (RS). Revista de Economia e Administração, São Paulo, v.11, n.3, 2012.

CRESWEL, J. W.. Projeto de Pesquisa: Métodos qualitativo, quantitativo e misto. 3 ed. Porto Alegre: Artmed, 2010.

CUFFNEY, T. F.; BRIGHTBILL, R. A.; MAY, J. T.; WAITE, I. R. Responses of Benthic Macroinvertebrates to Urbanization in Nine Metropolitan Areas. Ecol. Appl., v.20, n.5, p.1384$1401,2010$.

DAVIES, P. J.; FINDLAY, S. J.; WRIGHT, I. A.; JONASSON, O. J.. Impact of urban development on aquatic macroinvertebrates in south eastern Australia: Degradation of in-stream habitats and comparison with non-urban streams. Aquat. Ecol, v.44, n.4, p.685-700, 2010.

DOMENICO, D.; KRUGER, S. D.; MAZZIONI, S.; ZANIN, A.; LUDWIG, M. B. D.. Índice de sustentabilidade ambiental na produção leiteira. Revista de Administração, Contabilidade e Economia, v.16, n.1, p.261-282, 2017.

GEIST, J.; HAWKINS, S. J.. Habitat recovery and restoration in aquatic ecosystems: current progress and future challenges. Aquat. Conserv. Mar. Freshw. Ecosyst., v.26, p.942-962, 2016.

GONZALO, C.; CAMARGO, J. A.. The impact of an industrial effluent on the water quality, submersed macrophytes and benthic macroinvertebrates in a dammed river of central spain. Chemosphere, v.93, n.6, 2013.

GOULART, M. D.; CALLISTO, M.. Bioindicadores de qualidade de água como ferramenta em estudos de impacto ambiental. Revista FAPAM, v.2, n.1, p.153-164, 2003.

HAUER, F. R.; LAMBERTI, G. A.. Methods in Stream Ecology. 3 ed. Cambridge: Academic Press., 2007.

ISERMEYER, F.; HEMME, T.; HOLZNER, J.. Analysis of international competitiveness of milk production in the framework of the Institute of International Farm Comparison Network (IFCN). Agricultural Economics, v.49, n.2, p.94-100, 2003.

KNECHTEL, M. R.. Metodologia da pesquisa em educação: uma abordagem teórico prática dialogada. Curitiba: Inter Saberes, 2014.

LEMOS, B. M. et al. Tecnologia, especialização regional e produtividade: um estudo da pecuária leiteira em Minas Gerais. Rev. Econ. Sociol. Rural, Brasília, v.41, n.3, 2003.

LI, Z.; WANG, J.; LIU, Z.; MENG, X.; HEINO, J.; JIANG, X.; XIONG, X.; JIANG, X.; XIE, Z.. Different responses of taxonomic and functional structures of stream macroinvertebrate communities to local stressors and regional factors in a subtropical biodiversity hotspot. Sci. Total Environ., v.10, n.655, p.1288-1300, 2019.

LIMA FILHO, R. R.; TORRES JÚNIOR, A. M.; LOPES, A.; AGUIAR, G. A. M.. Mais um Ano do Dólar. Agroanalysis, São Luís, v.36, n.2, 2016.

LOPES, M. A.; REIS, E. M. B.; DEMEU, F. A.; MESQUITA, A. A.; ROCHA, A. G. F.; BENEDICTO, G. C.. Uso de ferramentas de gestão na atividade leiteira: um estudo de caso no sul de Minas Gerais. Rev. Cient. Prod. Anim., v.18, n.1, p.26-44, 2016.

MANGADZE, T.; BERE, T.; MWEDZI, T.. Choice of biota in stream assessment and monitoring programs in tropical streams: a comparison of diatoms, macroinvertebrates and fish. Ecol. Indic., v.63, p.128-143, 2016.

MENEGHATTI, R. M.; FARIÑA, L. O.; BERTOLINI, G. R. F.. Relação entre a cooperativa e cooperado na agricultura familiar: a busca pela sustentabilidade econômica dos produtores de leite. Revista Metropolitana de Sustentabilidade, v.7, n.1, 2017.

MEYER, M. D.; DAVIS, C.; DVORETT, D.. Response of Wetland Invertebrate Communities to Local and Landscape Factors in North Central Oklahoma. Wetlands, v.35, n.3,2015.

MORAES, M. M. B.; BENDER FILHO, R.. Mercado Brasileiro de Lácteos: análise do impacto de políticas de estímulo à 
produção. Rev. Econ. Sociol. Rural, v.55, n.4, 2003.

MORENO, P.; FRANÇA, J. S.; FERREIRA, W.; PAZ, A.. Use of the BEAST model for biomonitoring water quality in a neotropical basin. Hydrobiologia, v.630, n.1, p.231-242, 2009.

MOURA-SILVA, M. S. G.; GRACIANO, T. S.; LOSEKANN, M. E.; LUIZ, A. J. B.. Assessment of benthic macroinvertebrates at Nile tilapia production using artificial substrate samplers. Brazilian Journal of Biology, v.76, n.3, 2016.

NERES, S. L.; LOURENÇO JÚNIOR, J. B.; SANTOS, M. A. S.; NORONHA, G. N.; BEZERRA, A. S.; SILVA, A. G. M.. Caracterização da pecuária leiteira no município de Tailândia, Estado do Pará, Brasil. Revista de Ciências Agrárias, v.60, n.3, 2018.

NERO, L. A.; MATTOS, M. R.; BELOTI, V.; BARROS, M. A F.; PINTO, J. P. A. N.; ANDRADE, N. J.; SILVA, W. P.; FRANCO, B. D. G. M.. Leite cru de quatro regiões leiteiras brasileiras: perspectivas de atendimento dos requisitos microbiológicos estabelecidos pela Instrução Normativa 51. Ciência e Tecnologia de Alimentos, v.25, n.1, 2005.

OLIVEIRA, L. F. T.; SILVA, S. P.. Mudanças institucionais e produção familiar na cadeia produtiva do leite no Oeste Catarinense. Rev. Econ. Sociol. Rural, Brasília, v.50, n.4, p.705-720, 2012.

PAIXÃO, M. G.; LOPES, M. A.; PINTO, S. M.; ABREU, L. R.. Impacto econômico da implantação das boas práticas agropecuárias relacionadas com a qualidade do leite. Revista Ceres, v.61, n.5, 2014.

RANGANATH, S.; HESSION, W. C.; THOMPSON, T. M.. Livestock exclusion influences on riparian vegetation, channel morphology, and benthic macroinvertebrate assemblages. J. Soil Water Conserv., v.64, n.1, 2009.

REMPEL, C.; ECKHARDT, R. R.; JASPER, A.; SCHULTZ, G.; HILGERT, Í. H.; BARDEN, J. E.. Proposta metodológica de avaliação da sustentabilidade ambiental de propriedades produtoras de leite. Tecno-Lógica, v.16, n.1, 2012.

RESH, V. H.; JACKSON, J. K.. Rapid assessment approaches to biomonitoring using benthic macroinvertebrates. In: ROSENBERG, D. M.; RESH, V. H.. Freshwater Biomonitoring and Benthic Macroinvertebrates. New York, 1993.

RIOS, S. L.; BAILEY, R. C.. Relationship between riparian vegetation and stream benthic communities at three spatial scales. Hydrobiologia, v.553, n.1, p.153-160, 2006.

RODRIGUES, G. S.; RODRIGUES, I. A.; TUPY, O.; CAMARGO, A. C.; NOVO, A. L. M.; BONADIO, L. F.; TOKUDA, F. S.; ANDRADE, E. F.; SHIOTA, C. M.; SILVA, R. A.. Avaliação sócioambiental da integração tecnológica Embrapa Pecuária Sudeste para produção leiteira na agricultura familiar. Agricultura São Paulo, São Paulo, v.53, n.2, 2006.

SANTOS, I. G. A.; RODRIGUES, G. G.. Colonização de macroinvertebrados bentônicos em detritos foliares em um riacho de primeira ordem na Floresta Atlântica do Nordeste Brasileiro. Revista Iheringia, v.105, n.1, 2015

SILVA, M. F.; PEREIRA, J. C.; GOMES, S. T.; NASCIF, C.; GOMES, A. P.. Avaliação dos indicadores zootécnicos e econômicos em sistemas de produção de leite. Revista de Política Agrícola, v.24, n.1, 2015.

SOUZA, B. L. J.; ALVES, A. F.; CULTI, N. N.. A pecuária leiteira e o risco de exclusão nos municípios de Peabiru e Quinta do Sol. Interações, Campo Grande, v.14, n.2, 2013.

TUNDISI, J. G.. Recursos hídricos no futuro: problemas e soluções. Estudos Avançados, v.22, n.63, 2008.

WILLIS, R.. Farming. Asia Pacific Viewpoint, v.42, n.1, p.5565, 2001.

YAMAGUCHI, L. C. T. et al. Produção de leite no Brasil nas três últimas décadas. In: GOMES, A. T. et al.. $\mathbf{O}$ agronegócio do leite no Brasil. Juiz de Fora: Embrapa, 2001. p.33-48.

A CBPC - Companhia Brasileira de Produção Científica (CNPJ: 11.221.422/0001-03) detém os direitos materiais desta publicação. Os direitos referem-se à publicação do trabalho em qualquer parte do mundo, incluindo os direitos às renovações, expansões e disseminações da contribuição, bem como outros direitos subsidiários. Todos os trabalhos publicados eletronicamente poderão posteriormente ser publicados em coletâneas impressas sob coordenação da Sustenere Publishing, da Companhia Brasileira de Produção Científica e seus parceiros autorizados. Os (as) autores (as) preservam os direitos autorais, mas não têm permissão para a publicação da contribuição em outro meio, impresso ou digital, em português ou em tradução. 